\title{
Dedo azul agudo idiopático no isquémico: síndrome de Achenbach. Presentación de un caso y revisión bibliográfica
}

\author{
Juan Ignacio Pérez Abdala, Javier Sánchez Saba, Ezequiel E. Zaidenberg, Gerardo Gallucci, Jorge G. Boretto, \\ Pablo De Carli, Franco L. De Cicco \\ Sector de Cirugía de Mano y Miembro Superior, Instituto de Ortopedia y Traumatología "Prof. Dr. Carlos E. Ottolenghi", \\ Hospital Italiano de Buenos Aires, Ciudad Autónoma de Buenos Aires, Argentina
}

\begin{abstract}
RESUMEN
El síndrome de Achenbach es un cuadro de baja incidencia y de etiología idiopática. Se caracteriza por la aparición aguda de coloración azul digital sin un episodio desencadenante, imitando a un cuadro isquémico, pero, al contrario, el síndrome de Achenbach se autolimita sin dejar secuelas. Se presenta una mujer de 75 años que consultó en la central de emergencias por dolor y cambio de coloración violácea del dedo índice de su mano derecha, de inicio súbito. Los estudios complementarios no aportaron información relevante. Se administró un tratamiento sintomático y se logró la resolución completa del cuadro. La anamnesis y el examen físico exhaustivo tienen un rol fundamental para sospechar este cuadro y los estudios complementarios se reservan para descartar otras enfermedades, si es necesario. Consideramos que conocer esta enfermedad y un abordaje correcto conducen a un diagnóstico apropiado.
\end{abstract}

Palabras clave: Síndrome de Achenbach; hematoma digital; dedo azul; hemorragia paroxística.

Nivel de Evidencia: IV

\section{Acute Non-Ischemic Idiopathic Blue Finger: Achenbach's Syndrome. Case Report and Literature Review}

\section{ABSTRACT}

Achenbach's Syndrome (AS) is a pathology with low incidence and its etiology is unknown. It is characterized by the acute appearance of blue coloration in the finger without a triggering event and might be confused with an ischemic event. However, AS is a self-limited disease without sequelae. This case report is about a 75-year-old woman who presented sudden onset pain and purple coloration in the index finger of her right hand. Diagnostic tests were unhelpful and did not provide relevant information. She received symptomatic treatment, achieving complete resolution. The anamnesis and an exhaustive physical examination play a primary role in the suspicion; leaving diagnostic tests for the exclusion of other pathologies when they are required. We believe that knowing about this pathology allows a correct approach leading to an appropriate diagnosis.

Keywords: Achenbach's syndrome; digital bruising; blue finger; paroxysmal haemorrhage.

Level of Evidence: IV

\section{INTRODUCCIÓN}

El síndrome de Achenbach, también llamado síndrome del dedo azul, hematoma digital paroxístico o dedo azul agudo idiopático, es una entidad poco descrita en la bibliografía ${ }^{1-6} \mathrm{Si}$ bien se comunica una incidencia baja, de hasta cinco casos por millón de habitantes, se considera que su diagnóstico podría estar subestimado., ${ }^{4,-9}$ Tiene una prevalencia más alta en la población femenina, de entre 40 y 60 años. ${ }^{27,10,11}$ Aunque su etiología es desconocida, se ha descrito que puede relacionarse con una hemorragia subcutánea espontánea y se han postulado diversas hipótesis de alteración del flujo vascular y de fragilidad capilar para explicar este fenómeno., ${ }^{2,4,5,7,9,12-20}$ Como sus características clínicas de presentación hacen sospechar un episodio isquémico, el conocimiento de este cuadro

Recibido el 9-8-2020. Aceptado luego de la evaluación el 28-12-2020 • Dr. JUAN IGNACIO PÉREZ ABDALA • ignacio.perez@ hospitalitaliano.org.ar 
debería estar más difundido, teniendo en cuenta que los pacientes acuden a la atención médica a través de diferentes especialidades, como dermatología, cirugía vascular, clínica médica, medicina familiar, cirugía plástica y ortopedia. Es importante conocer esta enfermedad principalmente para evitar estudios de diagnóstico costosos e invasivos y poder ofrecer un tratamiento y un seguimiento adecuados. . $^{911,13,21}$

El objetivo de este artículo es presentar un caso de síndrome de Achenbach y una revisión bibliográfica, proponiendo una ruta diagnóstica sencilla y segura.

\section{CASO CLÍNICO}

Mujer de 75 años, jubilada, y de mano dominante derecha. Relataba antecedentes de polimialgia reumática, hipotiroidismo, depresión, dislipemia y gastritis crónica. Tomaba aspirina $100 \mathrm{mg} /$ día. Concurrió al Departamento de Urgencia de ortopedia por la aparición súbita de color violáceo en el dedo índice de su mano derecha. Refirió que, dos horas antes, había comenzado con dolor y edema de forma espontánea. A los pocos minutos, notó cambios de coloración en su dedo, se tornó violáceo, acompañado de sensación de calor y limitación funcional. No refirió una causa desencadenante, negó un traumatismo y la exposición a cambios bruscos de temperatura. Tampoco había sufrido episodios similares antes.

En el examen físico, se observó una coloración violácea de la región volar del dedo índice, sin compromiso de la falange distal. El diámetro estaba aumentado comparado con el contralateral y la flexión estaba limitada por el edema (Figura 1).
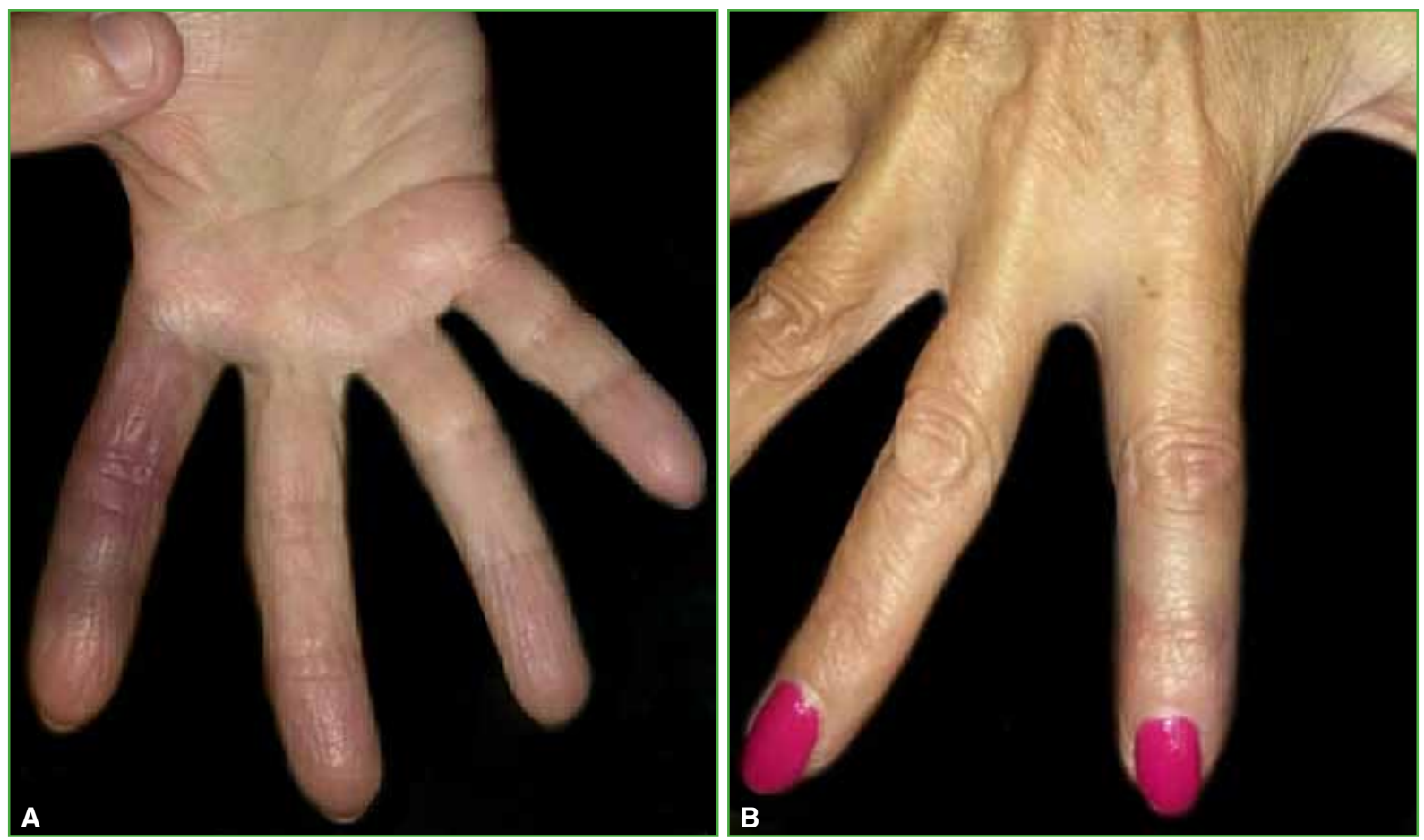

Figura 1. Imagen clínica de la mano derecha. A. Vista palmar. B. Vista dorsal.

Se constató lo siguiente: un relleno capilar $<2$ segundos, una adecuada temperatura local, pulsos periféricos radial y cubital, prueba de Allen en la muñeca y a nivel digital negativa. La sensibilidad estaba conservada, con discriminación a dos puntos $<6 \mathrm{~mm}$. Se evaluaron el dolor y la funcionalidad mediante la escala analógica visual $(0=\sin$ dolor; $10=$ el peor dolor $)$ y el puntaje DASH $(0=$ mejor resultado, $100=$ peor resultado $)$. El puntaje para el dolor era 5/10 en reposo y 7/10 en actividad, y el puntaje DASH inicial era de 63,75. Las radiografías no revelaron compromiso óseo (Figura 2). 


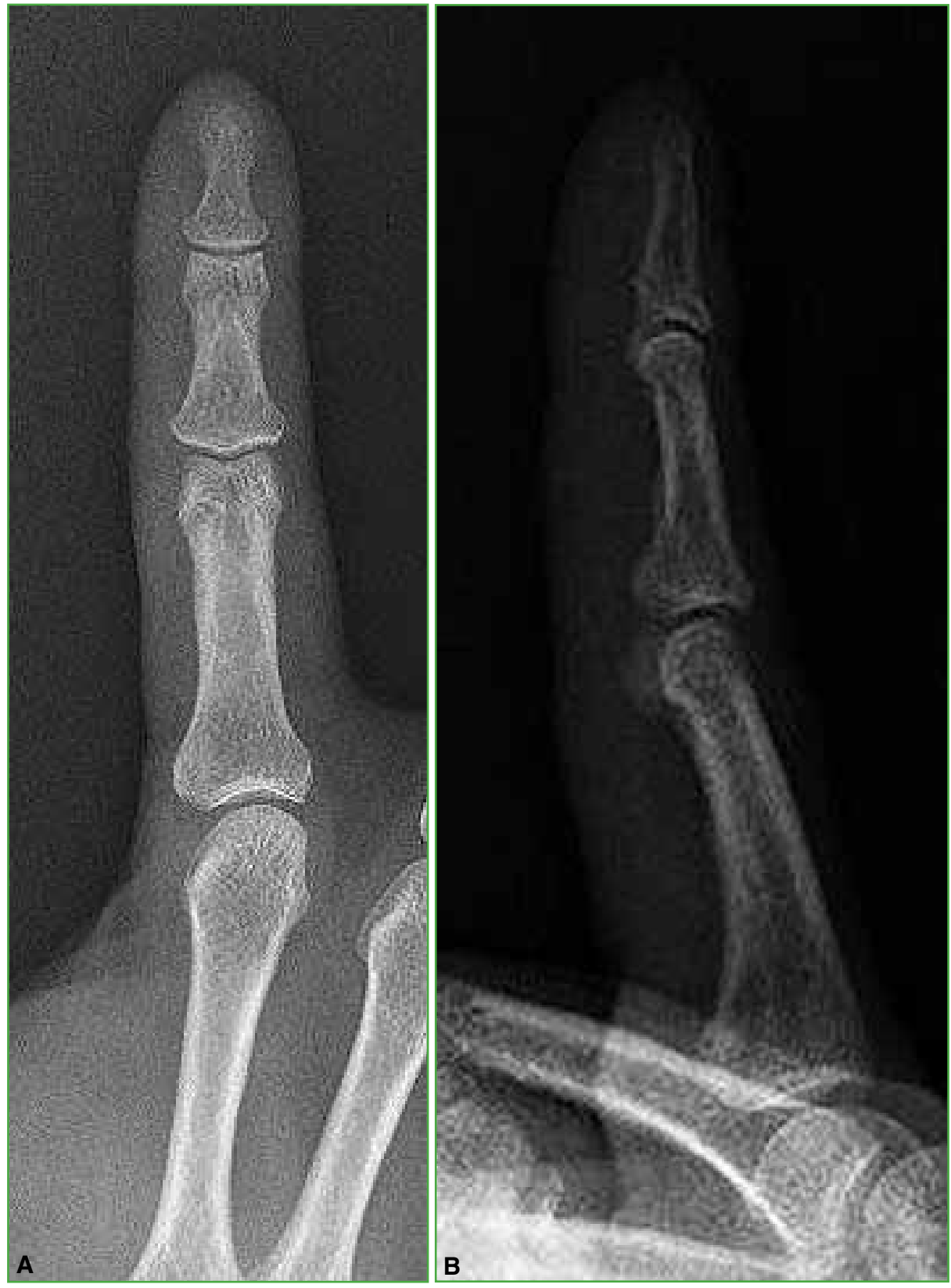

Figura 2. Radiografía del dedo índice derecho. A. De frente. B. De perfil.

A su vez, ante el compromiso de partes blandas, se solicitaron una ecografía y una ecografía Doppler de forma urgente, que mostraron edema subcutáneo, flujo arterial conservado y ausencia de trombosis. Se realizaron análisis bioquímicos que incluyeron hemograma, coagulograma (recuento de plaquetas, tiempo de protrombina, tiempo de tromboplastina parcial activado), ionograma, eritrosedimentación, proteína $\mathrm{C}$ reactiva y perfil lipídico. Todos estos valores estaban dentro de los parámetros normales. Teniendo en cuenta tanto la evaluación clínica como los exámenes complementarios, se indicó reposo manual y analgesia por vía oral, según necesidad. 
Durante los sucesivos controles, se observó una disminución progresiva de los signos clínicos, el cuadro se curó a las dos semanas, sin dejar secuelas, y tampoco hubo recurrencias hasta el alta definitiva a los cuatro meses del comienzo (Figura 3). En ese momento, la evaluación arrojó un puntaje de 0/10 tanto en reposo como en actividad, para la escala analógica visual, y un puntaje DASH de 16,5.
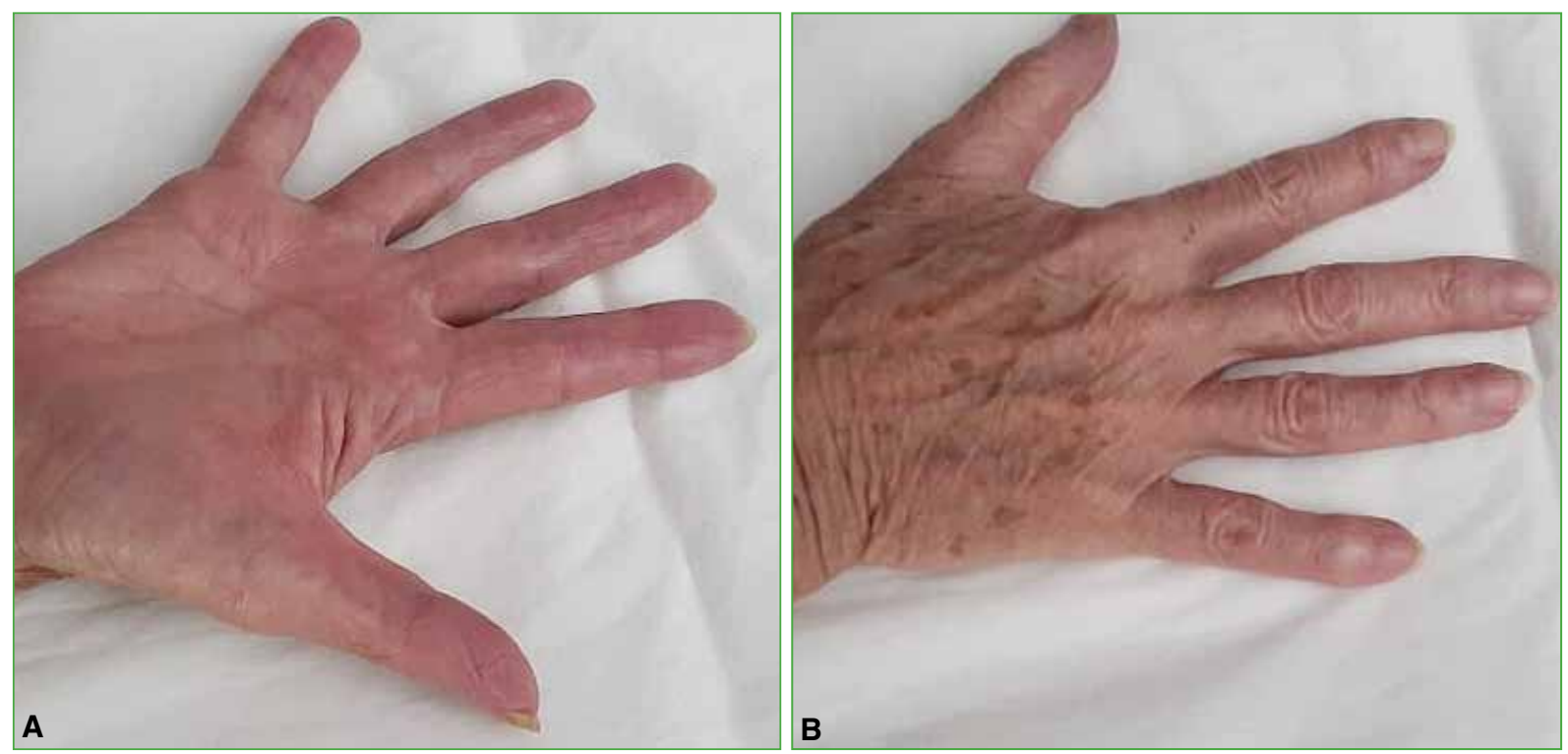

Figura 3. Imagen clínica de la mano derecha. A. Vista palmar. B. Vista dorsal.

\section{DISCUSIÓN}

El primer registro de esta enfermedad se remonta a 1958, por Walter Achenbach..$^{22}$ Es más prevalente en etnias de raza blanca, el sexo femenino (90\%) y durante la mediana edad. ${ }^{3,4,7,10,23}$ A pesar de que la etiología no es clara, Robertson y cols. asocian esta enfermedad a una reducción del flujo digital demostrada por angiografía; pero, por el contrario, Takeuchi y cols. no comunican cambios en el flujo digital. 3,17 Mediante el uso de la capilaroscopia, se observaron hemorragias, sin cambios morfológicos en los capilares; mientras que, por otro lado, se postuló la fragilidad de los pequeños vasos, posiblemente relacionada con la edad y el factor hormonal. , $^{8,16,18}$ Además, Kämpfen y cols. publicaron que el uso de la ergotamina puede provocar una reacción vasoespástica. ${ }^{12}$ Como aseguran los numerosos estudios, no sería una expresión de una enfermedad sistémica, pero se la asocia con artritis reumatoide, tiroiditis autoinmune, enfermedad gastrointestinal y de la vesícula biliar, acrocianosis y migraña. ${ }^{1,14,24}$

Su presentación clínica consiste en la aparición súbita de cambio de coloración azul-violácea, frecuentemente precedida por síntomas prodrómicos, como picazón, molestias, parestesias o dolor. ${ }^{24,25}$ Luego del cambio de coloración, que puede virar a pálido o rojizo, los síntomas que acompañan al cuadro son dolor (60-100\%), edema (60\%), parestesias (20-40\%), limitación funcional y, en menor medida, sensación de calor o frialdad. $7,10,11,14,17,23,24$ El cambio de coloración es explicado, tanto por histología como por los estudios por imágenes, como un mecanismo de hemorragia subcutánea, sin transitar por los estadios que comúnmente un hematoma atraviesa. ${ }^{1,3,4,15,16,19}$ Se considera de aparición espontánea, aunque algunos reportes lo asocian a fenómenos mecánicos, como traumas menores o efectos cizallantes durante actividades repetitivas con la mano afectada, y la exposición solar. ${ }^{3,7,11,16,19}$

Las regiones más afectadas son las manos, con predominio del lado derecho, y los dedos índice y mayor, aunque puede presentarse en otras áreas, como los pies. ${ }^{2,7,10,11,15,24}$ Es característica la ausencia de compromiso de la falange distal y su delimitación a la región volar, aunque no es un signo patognomónico. ${ }^{2,4,21}$ Según algunos autores, hay riesgo de recurrencia (un promedio de hasta 3 episodios) y, en otras publicaciones, se asoció la presencia de algún familiar con antecedente de síndrome de Achenbach como un factor predisponente. ${ }^{7,9,23}$ El tratamiento es 
puramente sintomático, con analgésicos, crioterapia, inmovilización y reposo. ${ }^{4,7}$ Su pronóstico es benigno, no deja secuelas, y resuelve espontáneamente a los pocos días de comenzar, aunque puede extenderse hasta seis semanas, según algunos autores. ${ }^{2,3,7,21}$

El examen físico es fundamental para valorar los distintos diagnósticos diferenciales. Se deben evaluar las zonas afectadas teniendo en cuenta lo siguiente: si hay compromiso de todo el dedo, se puede sospechar un episodio isquémico o síndrome de Raynaud; si están afectadas las zonas acrales, sospechar acrocianosis, acrorrigosis o eritema pernio; y si aparece en regiones distintas de las acrales, puede considerarse púrpura psicogénica (por estrés emocional) o dermatitis artefacta. ${ }^{7,21} \mathrm{El}$ dedo comprometido puede estar frío en el episodio isquémico o el síndrome de Raynaud, o caliente en la eritromegalia. ${ }^{21}$ Se observan estigmas cutáneos, como úlceras o gangrena, en la tromboangeítis obliterante y la presencia de nódulos (trombos) en la trombosis de venas digitales. ${ }^{7,21}$ La palpación de los pulsos periféricos y la prueba de Allen, tanto en la muñeca como digital de forma bilateral, son útiles ante la sospecha de un episodio isquémico de origen embólico, trombótico, vasoespástico o vasculítico. ${ }^{11}$ La prueba de Adson suele ser positiva en un síndrome del opérculo torácico. ${ }^{7,21}$ Finalmente, no debe desestimarse la presencia de síntomas y signos sistémicos que puedan expresar colagenopatías o endocarditis bacteriana. ${ }^{7,11,21}$

Los métodos complementarios empleados, como se ha comprobado, no aportan datos específicos positivos para el diagnóstico del síndrome de Achenbach y su utilidad radica en descartar los diagnósticos diferenciales. ${ }^{2,4,7,24}$ Estos incluyen la toma de la presión arterial con índice tobillo/brazo, saturometría con oxímetro de pulso, ecografía convencional, y ecografía Doppler venosa y arterial; análisis de laboratorio, como hemograma, coagulograma, razón internacional normatizada, ionograma, función renal y hepática, eritrosedimentación, proteína $\mathrm{C}$ reactiva, perfil lipídico e inmunológico (anticuerpos antinucleares, factor reumatoide, anti-ADN) y serología viral, radiografías, resonancia magnética, ecocardiograma, angiotomografía o angiografía, capilaroscopia, y hasta la toma de biopsia. ${ }^{3,9,12,16-19,21} \mathrm{Si}$ es necesario, se debería comenzar con métodos incruentos que incluyan análisis completos, radiografía y ecografía Doppler que ayudarán a descartar lesiones traumáticas, trastornos inmunológicos y vasculares; y avanzar a estudios más invasivos cuando no se haya podido establecer el diagnóstico, como angiografía o angiotomografía, que pueden ser útiles tanto para el diagnóstico como para un eventual tratamiento.

Consideramos que la falta de conocimiento sobre el síndrome de Achenbach y la creencia de que represente un cuadro de urgencia revelan la importancia de su conocimiento. Por esto, entendemos que una exhaustiva anamnesis y un correcto examen físico serían suficientes para su diagnóstico y, por lo tanto, los exámenes complementarios quedarían reservados para aquellos casos de duda diagnóstica.

Conflicto de intereses: Los autores no declaran conflictos de intereses.

ORCID de J. Sánchez Saba: https://orcid.org/0000-0001-5496-3513 ORCID de E. E. Zaidenberg: https://orcid.org/0000-0002-1535-0586 ORCID de G. Gallucci: https://orcid.org/0000-0002-0612-320X
ORCID de J. G. Boretto: https://orcid.org/0000-0001-7701-3852

ORCID de P. De Carli: https://orcid. org/0000-0002-9474-8129

ORCID de F. L. De Cicco: https://orcid.org/0000-0001-9844-140X

\section{BIBLIOGRAFÍA}

1. Manappallil RG, Jayaraj J. Blue finger syndrome: an unusual presentation of rheumatoid arthritis. J Clin Diagn Res 2017;11(5):OD06-7. https://doi.org/10.7860/JCDR/2017/25300.9784

2. Godoy A, Tabares AH. Achenbach syndrome (paroxysmal finger hematoma). Vasc Med 2019;24(4):361-6. https://doi.org/10.1177/1358863X19849627

3. Takeuchi H, Uchida HA, Okuyama Y, Wada J. Acute idiopathic blue fingers: a young man with Achenbach's syndrome. BMJ Case Rep 2016;2016:101136/bcr2016214491. https://doi.org/10.1136/bcr-2016-214491

4. Harper CM, Waters PM. Acute idiopathic blue finger: case report. J Hand Surg Am 2013;38(10):1980-2. https://doi.org/10.1016/j.jhsa.2013.07.022 
5. Hayta SB, Guner R. A case with acute idiopathic blue finger. Cumhuriyet Medical Journal 2017;39(3):635-6. https://doi.org/10.7197/223.v39i31705.347465

6. Sigha B, Josselin L, Gatfosse M, Fardet L. Hématomes digitaux spontanés paroxystiques (syndrome d'Achenbach). Ann Dermat Vénér 2016;143(2):130-3. https://doi.org/10.1016/j.annder.2015.10.597

7. Ada F, Kasimzade F. Analysis of 24 patients with Achenbach's syndrome. World J Clin Cases 2019:7(10):1103-10. https://doi.org/10.12998/wjcc.v7.i10.1103

8. Carpentier PH, Maricq HR, Biro C, Jiguet M, Seinturier C. Paroxysmal finger haematoma - a benign acrosyndrome occurring in middle-aged women. Vasa 2016;45(1):57-62. https://doi.org/10.1024/0301-1526/a000496

9. Seifman MA, Johnstone BR. The acutely blue finger: cause for concern? J Hand Surg Asian Pac 2018;23(2):294-6. https://doi.org/10.1142/s2424835518720219

10. Jaimes YAP, Chinome JEO, Molina-Franky J. Hematoma digital espontáneo, síndrome de Achenbach. Revista de la Facultad de Ciencias Médicas de Córdoba 2019;76(4):257-60. https://doi.org/10.31053/1853.0605.v76.n4.24963

11. Kordzadeh A, Caine PL, Jonas A, Rhodes KM, Panayiotopolous YP. Is Achenbach's syndrome a surgical emergency? A systematic review. Eur J Trauma Emerg Surg 2016;42(4):439-43. https://doi.org/10.1007/s00068-015-0610-0

12. Kämpfen S, Santa DD, Fusetti C. A Painful blue thumb: a case of Achenbach's syndrome. EJVES Extra 2005;10(4):84-5. https://doi.org/10.1016/j.ejvsextra.2005.07.004

13. Weinberg I, Jaff MR. Spontaneous blue finger syndrome: a benign process. Am J Med 2012;125(1):e1-2. https://doi.org/10.1016/j.amjmed.2011.05.007

14. Ribeiro F, Aveiro M, Leal M, Valente T, Jesus G. An acute blue finger: a case of Achenbach's syndrome. Eur J Case Rep Intern Med 2019;6(9):001231. https://doi.org/10.12890/2019_001231

15. Pavlovic MD, Loubser MD. Paroxysmal acral haematoma is a more appropriate name for Achenbach syndrome. Clin Exp Dermatol 2019;44(2):e18-9. https://doi.org/10.1111/ced.13836

16. Huikeshoven M, de Priester JA, Engel AF. A case of spontaneous wrist haematoma in Achenbach syndrome. $J$ Hand Surg Eur 2009;34(4):551-2. https://doi.org/10.1177/1753193409103731

17. Robertson A, Liddington MI, Kay SP. Paroxysmal finger haematomas (Achenbach's syndrome) with angiographic abnormalities. J Hand Surg Br 2002;27(4):391-3. https://doi.org/10.1054/jhsb.2001.0726

18. Frerix M, Richter K, Müller-Ladner U, Hermann W. Achenbach's syndrome (paroxysmal finger hematoma) with capillaroscopic evidence of microhemorrhages. Arthritis Rheumatol 2015;67(4):1073. https://doi.org/10.1002/art.39003

19. Watchorn RE, Babu S, Lewis F, Calonje E, Taibjee SM. Paroxysmal purple palmar macules with a rare aetiology. Clin Exp Dermatol 2017;42(5):561-3. https://doi.org/10.1111/ced.13101

20. Eikenboom JCJ, Cannegieter SC, Briët E. Paroxysmal finger haematoma: a neglected syndrome. Thromb Haemost 1991;66(2):266. http://doi.org/10.1055/s-0038-1646403

21. Khaira HS, Rittoo D, Vohra RK, Smith SR. The non-ischaemic blue finger. Ann R Coll Surg Engl 2001;83(3):154-7. PMID: 11432130.

22. Achenbach W. [Paroxysmal hematoma of the hand]. Medizinische 1958;52(27):2138-40. PMID: 13622116

23. Yamada T. Achenbach's syndrome in an elderly woman. J Gen Fam Med 2018;19:65-6. https://doi.org/10.1002/jgf2.158

24. Jiménez PR, Ocampo MI, Castañeda-Cardona C, Rosselli D. Achenbach's syndrome: Case report and systematic review of the literature. Rev Colomb Reumatol (English Edition) 2017;24(4):230-6. https://doi.org/10.1016/j.rcreue.2018.04.003

25. Suzuki J. Achenbach's syndrome in a 30-year-old healthy woman. Intern Med 2019;58(12):1807. https://doi.org/10.2169/internalmedicine.2263-18 